\title{
Spacetime mappings of the Brown-York quasilocal energy
}

\author{
Jeremy Côtéa , Marianne Lapierre-Léonard ${ }^{\mathrm{b}}$, Valerio Faraonic \\ Department of Physics and Astronomy, Bishop's University, 2600 College Street, Sherbrooke, QC J1M 1Z7, Canada
}

Received: 23 May 2019 / Accepted: 6 August 2019 / Published online: 13 August 2019

(c) The Author(s) 2019

\begin{abstract}
In several areas of theoretical physics it is useful to know how a quasilocal energy transforms under conformal rescalings or generalized Kerr-Schild mappings. We derive the transformation properties of the Brown-York quasilocal energy in spherical symmetry and we contrast them with those of the Misner-Sharp-Hernandez energy.
\end{abstract}

\section{Introduction}

The mass of a non-isolated system in General Relativity (GR) has been the subject of intense study but there is no agreement as to what the mass-energy should be. Due to the equivalence principle, the energy of the gravitational field cannot be localized and the mass-energy of a self-gravitating system includes also this energy. Unless the geometry reduces asymptotically to Minkowski (in which case the ADM energy [1] is appropriate), one resorts to quasilocal energy definitions. There are several quasilocal constructs in the literature, which differ from each other (see [2] for a recent review). Overall, quasilocal energy has been studied in the domain of formal relativity, but one ought to do better. First, the mass of a gravitating system is one of its most basic properties in astrophysics and a mass-energy definition is ultimately of no use if it cannot be employed in practical calculations (for example, in astrophysics and/or in cosmology). Second, various authors are already using, implicitly, the Hawking-Hayward quasilocal energy $[3,4]$ (usually in its Misner-Sharp-Hernandez form defined for spherical symmetry [5,6]) in black hole thermodynamics [7-18], in which this quasilocal energy plays the role of the internal energy of the system.

Black hole thermodynamics (especially the thermodynamics of time-dependent apparent horizons) is usually stud-

\footnotetext{
a e-mail: jcote16@ubishops.ca

be-mail: mlapierre12@ubishops.ca

c e-mail: vfaraoni@ubishops.ca
}

ied in the context of GR and most often in spherical symmetry, where the Misner-Sharp-Hernandez mass is adopted almost universally [7-18] (see, however, Ref. [19] for an analogous study using the Brown-York mass). The MisnerSharp-Hernandez mass is also the quasilocal construct used in spherical fluid dynamics and in black hole collapse [5,6] and is the Noether charge associated with the covariant conservation of the Kodama energy current [52]. But there are several other definitions of quasilocal energy [2] and one wonders what changes the use of another quasilocal construct, for example the Brown-York energy, would bring. When a black hole is dynamical, it is difficult to calculate its temperature unambiguously and the recent literature on the thermodynamics of dynamical black holes focuses on this quantity. If the definition of internal energy is also uncertain, the problems accumulate. Quasilocal energies have been used also in the now rather broad field of thermodynamics of spacetime [20-36].

A full discussion of which quasilocal mass should be used, and why, requires more insight on quasilocal energies than is presently available. Here we consider a particular aspect, more related to tool-building than to core issues, which has been discussed recently in the literature. Since several analytic solutions of the Einstein field equations which describe dynamical black holes are generated by using the Schwarzschild (or another static black hole) solution as a seed and performing a conformal or a Kerr-Schild transformation [37-50], the transformation properties of the Misner-Sharp-Hernandez mass under these spacetime mappings were discussed [51]. Later, relinquishing the simplifying assumption of spherical symmetry, the transformation properties of the Hawking-Hayward quasilocal energy [3,4] (which reduces to the Misner-Sharp-Hernandez prescription $[5,6]$ in spherical symmetry [52]) were also obtained [53]. Generalizations of the Hawking-Hayward energy to scalartensor gravity have also been introduced ([54-57], see also [58-62], and [63,64] for the case of Lovelock gravity), following earlier generalizations of the Brown-York mass to 
these theories [65-67]. A useful trick consists of remembering that these theories admit a representation in the Einstein conformal frame which is formally very similar to GR. If one chooses a different quasilocal energy, it becomes important to establish how this construct transforms under these spacetime mappings.

There are also other motivations for studying the transformation properties of quasilocal energies. As noted above, these quantities are defined rather formally and are not yet used in practical calculations in astrophysics and cosmology, with the exception of the recent works [68-70]. There, the Hawking-Hayward quasilocal construction was employed in a new approach to cosmological problems in which the expansion of the universe competes with the local dynamics of inhomogeneities, namely the Newtonian simulations of large scale structure formation in the early universe [68], the turnaround radius in the present accelerated universe [69], and lensing by the cosmological constant or by dark energy [70]. To first order in the metric perturbations present in these problems, the Brown-York energy yields the same results as the Hawking-Hayward energy, provided that an appropriate gauge is chosen for the gauge-dependent Brown-York energy in the comparison [71]. Conformal transformations were used in these works as a mere calculational tool, not for any conceptual reason. This is one more reason to establish how the Brown-York mass behaves under spacetime mappings, if it was going to replace the Hawking-Hayward/MisnerSharp-Hernandez construct.

In this work we restrict to spherical symmetry and, correspondingly, to a line element expressed in the gauge ${ }^{1}$

$d s^{2}=-A(t, R) d t^{2}+B(t, R) d R^{2}+R^{2} d \Omega_{(2)}^{2}$,

where $R$ is the areal radius, a well-defined geometric invariant once spherical symmetry is assumed, and $d \Omega_{(2)}^{2} \equiv$ $d \theta^{2}+\sin ^{2} \theta d \varphi^{2}$ is the line element on the unit 2-sphere. It is well known that, in this gauge, the Brown-York mass is given by [73-75]

$M_{B Y}=R\left(1-\frac{1}{\sqrt{B}}\right)$.

The general definition of Brown-York mass is based on an integral of the extrinsic curvature of a 3-surface in the real space minus the same quantity evaluated on the same Riemannian surface but with a Riemannian 3-space as a reference [73]. It is clear from the definition that the Brown-York mass is gauge-dependent. By contrast, the Misner-SharpHernandez mass $M_{M S H}$ is given by the scalar equation

$1-\frac{2 M_{M S H}(R)}{R}=\nabla^{c} R \nabla_{c} R$

${ }^{1}$ We follow the notations of Ref. [72]. and is, therefore, gauge-independent, which is a significant practical advantage over the Brown-York mass.

Since the Brown-York mass is gauge-dependent, it makes sense to derive its transformation properties under conformal and Kerr-Schild spacetime mappings only when a certain gauge is preserved by the map. This is what we do in the following sections. Since both the expression and the value of the Brown-York mass are very different in different gauges, it is meaningless to compare them in these different gauges.

\section{Conformal transformations}

A conformal transformation of the metric is the pointdependent rescaling

$g_{a b} \rightarrow \tilde{g}_{a b}=\Omega^{2} g_{a b}$,

where the conformal factor $\Omega$ is a smooth positive function of the spacetime point. We require the conformal factor to respect the spherical symmetry, $\Omega=\Omega(t, R)$. Under such a mapping, the line element (1) becomes

$d \tilde{s}^{2}=\Omega^{2} d s^{2}=-\Omega^{2} A d t^{2}+\Omega^{2} B d R^{2}+\tilde{R}^{2} d \Omega_{(2)}^{2}$,

where the "new" areal radius is $\tilde{R}=\Omega R$. The line element (5) is not in the form (1). To bring it to this form with tilded quantities, i.e.,

$d \tilde{s}^{2}=-\tilde{A} d \tilde{t}^{2}+\tilde{B} d \tilde{R}^{2}+\tilde{R}^{2} d \Omega_{(2)}^{2}$,

one has to introduce a new time coordinate. Begin by substituting the differential

$d R=\frac{d \tilde{R}-\Omega_{, t} R d t}{\Omega_{, R} R+\Omega}$

in the line element (1), obtaining

$$
\begin{aligned}
d \tilde{s}^{2}= & -\Omega^{2}\left[A-\frac{\Omega_{, t}^{2} R^{2} B}{\left(\Omega_{, R} R+\Omega\right)^{2}}\right] d t^{2} \\
& +\frac{\Omega^{2} B}{\left(\Omega_{, R} R+\Omega\right)^{2}} d \tilde{R}^{2} \\
& -\frac{2 \Omega^{2} \Omega_{, t} B R}{\left(\Omega_{, R} R+\Omega\right)^{2}} d t d \tilde{R}+\tilde{R}^{2} d \Omega_{(2)}^{2} .
\end{aligned}
$$

In order to bring this line element back to the gauge (1), one eliminates the term proportional to $d t d \tilde{R}$ by changing the time coordinate to $\tilde{t}(t, \tilde{R})$ defined by

$d \tilde{t}=\frac{1}{F}(d t+\beta d \tilde{R})$,

where $\beta(t, R)$ is a function to be determined in such a way that the $d t d \tilde{R}$ term disappears and $F(t, \tilde{R})$ is a (non-unique) 
integrating factor satisfying

$$
\frac{\partial}{\partial \tilde{R}}\left(\frac{1}{F}\right)=\frac{\partial}{\partial t}\left(\frac{\beta}{F}\right)
$$

to guarantee that $d \tilde{t}$ is an exact differential. Using $d t=$ $F d \tilde{t}-\beta d \tilde{R}$ in the line element gives

$$
\begin{aligned}
d \tilde{s}^{2}= & -\Omega^{2}\left[A-\frac{\Omega_{, t}^{2} R^{2} B}{\left(\Omega_{, R} R+\Omega\right)^{2}}\right] F^{2} d \tilde{t}^{2} \\
& +\left\{-\beta^{2}\left[\Omega^{2} A-\frac{\Omega_{, t}^{2} R^{2} \Omega^{2} B}{\left(\Omega_{, R} R+\Omega\right)^{2}}\right]+\frac{\Omega^{2} B}{\left(\Omega_{, R} R+\Omega\right)^{2}}\right. \\
& \left.+\frac{2 \beta \Omega_{, t} \Omega^{2} B R}{\left(\Omega_{, R} R+\Omega\right)^{2}}\right\} d \tilde{R}^{2}+2 F \Omega^{2} . \\
& +\left\{\beta\left[A-\frac{\Omega_{, t}^{2} R^{2} B}{\left(\Omega_{, R} R+\Omega\right)^{2}}\right]-\frac{\Omega_{, t} R B}{\left(\Omega_{, R} R+\Omega\right)^{2}}\right\} d \tilde{t} d \tilde{R} \\
& +\tilde{R}^{2} d \Omega_{2}^{2} .
\end{aligned}
$$

By imposing that

$$
\beta(t, R)=\frac{\Omega_{, t} B R}{\left[A-\frac{\Omega_{, t}^{2} R^{2} B}{\left(\Omega_{, R} R+\Omega\right)^{2}}\right]\left(\Omega_{, R} R+\Omega\right)^{2}}
$$

the line element becomes

$$
\begin{aligned}
d \tilde{s}^{2}= & -\Omega^{2}\left[A-\frac{\Omega_{, t}^{2} B R^{2}}{\left(\Omega_{, R} R+\Omega\right)^{2}}\right] F^{2} d \tilde{t}^{2} \\
& +\frac{A B \Omega^{2}}{A\left(\Omega_{, R} R+\Omega\right)^{2}-\Omega_{, t}^{2} B R^{2}} d \tilde{R}^{2}+\tilde{R}^{2} d \Omega_{2}^{2} .
\end{aligned}
$$

Therefore, we have

$$
\tilde{B}=\frac{A B \Omega^{2}}{A\left(\Omega_{, R} R+\Omega\right)^{2}-\Omega_{, t}^{2} B R^{2}} .
$$

Using this expression, Eq. (2) gives the Brown-York mass in the conformally rescaled world and in the chosen gauge

$\tilde{M}_{B Y}=\Omega R\left[1-\frac{\sqrt{A\left(\Omega_{, R} R+\Omega\right)^{2}-\Omega_{, t}^{2} B R^{2}}}{\sqrt{A B} \Omega}\right]$.

In general, there is no simple expression of the "new" Brown-York mass in terms of the "old" one plus a simple correction, analogous to the one previously obtained for the Misner-Sharp-Hernandez mass [51]. One could, for example, rewrite Eq. (15) as

$$
\begin{aligned}
& \tilde{M}_{B Y}=\Omega M_{B Y}+\frac{R}{\sqrt{A B}} \\
& \times\left(\Omega \sqrt{A}-\sqrt{A(\Omega, R R+\Omega)^{2}-\Omega_{, t}^{2} B R^{2}}\right)
\end{aligned}
$$

but this decomposition is arbitrary and not particularly enlightening anyway, even in the simplest situations in which the scale factor depends only on one of the variables $(t, R)$. For comparison, the transformation property of the MisnerSharp-Hernandez mass under a conformal rescaling is [51]

$\tilde{M}_{M S H}=\Omega M_{M S H}-\frac{R^{3}}{2 \Omega} \nabla^{a} \Omega \nabla_{a} \Omega-R^{2} \nabla^{a} \Omega \nabla_{a} R$.

The first term $\Omega M_{B Y}$ in Eq. (16) can be interpreted by introducing Newton's constant and remembering that, in a simple intepretation (dating back to Dicke) lengths and times scale with $\Omega$, while masses scale with $\Omega^{-1}$ [77]. However, the quasilocal energy is a complicated construct and cannot be expected to scale in a simple way under conformal transformations. As a consequence, the second term in the right hand side of Eq. (16) defies simple physical interpretation (the same can be said for the transformation property (17) of the Misner-Sharp-Hernandez mass).

The effect of the transformation (16) on black hole thermodynamics is difficult to interpret. A Smarr relation was derived in Ref. [19] for vacuum, static, spherical black holes of the form (1):

$2 T S=M_{B Y}+2 p A$,

where $T$ and $S$ are the temperature and area of the event horizon, $S$ is the entropy, and

$p=\frac{1}{8 \pi}\left(\frac{A_{H}^{\prime}}{2 A_{H} \sqrt{B_{H}}}+\frac{1}{r_{H} \sqrt{B_{H}}}-\frac{1}{r_{H}}\right)$,

while the subscript $H$ denotes quantities evaluated at the horizon. Assuming that, under a conformal transformation, $\tilde{T} \simeq T / \Omega$ in an adiabatic approximation (as argued in [39, 78]), $S=A / 4$, and $\tilde{A}=\Omega^{2} A$, Eq. (19) would yield

$$
\begin{aligned}
2 \tilde{T} \tilde{S}= & \tilde{M}_{B Y}-\frac{R}{\sqrt{A B}} \\
& \times\left(\Omega \sqrt{A}-\sqrt{A\left(\Omega_{, R} R+\Omega\right)^{2}-\Omega_{, t} B R^{2}}\right) \\
& +\frac{2 p \tilde{A}}{\Omega}
\end{aligned}
$$

in the tilded world. Not much should be construed from this complicated relation between tilded quantities: a conformal transformation with $\Omega=\Omega(t, r)$ preserving the spherical symmetry has changed the situation in which Eq. (19) was derived [19]. Vacuum is no longer vacuum and, if $\Omega_{, t} \neq 0$, the black hole is not static, the event horizon is no longer present, and the notion of black hole is now defined by an apparent (instead of event) horizon, which is not null [79]. The time dependence of the (apparent) horizon must be taken into account even in an adiabatic approximation [39,78]. Therefore, simple statements on the effect of the conformal transformation on thermodynamics cannot be made. 


\section{Kerr-Schild transformations}

A generalized Kerr-Schild transformation has the form

$g_{a b} \rightarrow \bar{g}_{a b}=g_{a b}+2 \lambda l_{a} l_{b}$,

where $\lambda$ is a positive function and $l^{a}$ is a null and geodesic vector field of $g_{a b}$, that is,

$g_{a b} l^{a} l^{b}=0, \quad l^{a} \nabla_{a} l^{b}=0$.

It is easy to see that $l^{a}$ is null and geodesic also with respect to $\bar{g}_{a b}$ :

$\bar{g}_{a b} l^{a} l^{b}=g_{a b} l^{a} l^{b}+2 \lambda\left(l_{c} l^{c}\right)^{2}=0$,

$\bar{g}_{a b} l^{a} \nabla^{b} l^{c}=0$,

and that the inverse metric of $\bar{g}_{a b}$ is $\bar{g}^{a b}=g^{a b}-2 \lambda l^{a} l^{b}$ since $\bar{g}^{\mu v} \bar{g}_{\nu \alpha}=\delta_{\alpha}^{\mu}$ [51]. In order to respect the spherical symmetry of the geometry (1) we require that $\lambda=\lambda(t, R)$ and

$l^{\mu}(t, R)=\left(l^{0}, l^{1}, 0,0\right)$

in this gauge. The generalized Kerr-Schild transformation (21) gives

$$
\begin{aligned}
d \bar{s}^{2}= & d s^{2}+2 \lambda l_{a} l_{b} d x^{a} d x^{b} \\
= & -\left[A-2 \lambda\left(l_{0}\right)^{2}\right] d t^{2} \\
& +\left[B+2 \lambda\left(l_{1}\right)^{2}\right] d R^{2}+4 \lambda l_{0} l_{1} d t d R \\
& +R^{2} d \Omega_{(2)}^{2} .
\end{aligned}
$$

We now repeat the procedure of Ref. [51] in order to eliminate the cross-term in $d t d R$. To this end, it is necessary to introduce a new time coordinate $T$ defined by

$d T=\frac{1}{F}(d t+\beta d R)$,

where $\beta(t, R)$ is a function to be determined and $F(t, R)$ is an integrating factor. The substitution of $d t=F d T-\beta d R$ into the line element yields

$$
\begin{aligned}
d \bar{s}^{2}= & -\left[A-2 \lambda\left(l_{0}\right)^{2}\right] F^{2} d T^{2} \\
& +\left\{B+2 \lambda\left(l_{1}\right)^{2}-\beta^{2}\left[A-2 \lambda\left(l_{0}\right)^{2}\right]-4 \lambda l_{0} l_{1} \beta\right\} d R^{2} \\
& +2 F\left\{\beta\left[A-2 \lambda\left(l_{0}\right)^{2}\right]+2 \lambda l_{0} l_{1}\right\} d T d R \\
& +R^{2} d \Omega_{(2)}^{2}
\end{aligned}
$$

from which one deduces that the required form of the function $\beta$ is

$\beta(t, R)=\frac{-2 \lambda l_{0} l_{1}}{A-2 \lambda\left(l_{0}\right)^{2}}$.
With this choice, the metric is brought back to the gauge (1),

$$
\begin{aligned}
d \bar{s}^{2}= & -\left[A-2 \lambda\left(l_{0}\right)^{2}\right] F^{2} d T^{2} \\
& +\left\{B+2 \lambda\left(l_{1}\right)^{2}+\frac{4 \lambda^{2}\left(l_{0}\right)^{2}\left(l_{1}\right)^{2}}{A-2 \lambda\left(l_{0}\right)^{2}}\right\} d R^{2} \\
& +R^{2} d \Omega_{(2)}^{2}
\end{aligned}
$$

where we note that

$\bar{B}=B+\frac{2 \lambda A\left(l_{1}\right)^{2}}{A-2 \lambda\left(l_{0}\right)^{2}}$

and there is residual gauge freedom due to the nonuniqueness of the integrating factor $F$. The Brown-York mass of the barred spacetime is then given by the expression (2) as

$\bar{M}_{B Y}=R\left(1-\frac{1}{\sqrt{B+\frac{2 \lambda A\left(l_{1}\right)^{2}}{A-2 \lambda\left(l_{0}\right)^{2}}}}\right)$.

Because of the normalization $l_{c} l^{c}=0$ of the null vector $l^{a}$, it is possible to rescale its components so that, say, $l_{0}=-1$. Then

$l^{\mu}=\left(\frac{1}{A}, \frac{ \pm 1}{\sqrt{A B}}, 0,0\right)$

and Eq. (32) simplifies to

$$
\begin{aligned}
\bar{M}_{B Y} & =R\left(1-\frac{\sqrt{A-2 \lambda}}{\sqrt{A B}}\right) \\
& =M_{B Y}+\frac{R}{\sqrt{B}}\left(1-\sqrt{1-\frac{2 \lambda}{A}}\right) .
\end{aligned}
$$

For comparison, the transformation property of the MisnerSharp-Hernandez mass under a generalized Kerr-Schild map is [51]

$\bar{M}_{M S H}=M_{M S H}+\frac{\lambda R}{A B}$.

Again, the action of the Kerr-Schild transformation arising from a nonvanishing $\lambda$ cannot be given a simple interpretation due to the fact that quasilocal energies are rather complicated constructs.

\section{Examples}

Here we present examples illustrating the transformation properties of the Brown-York mass. 


\subsection{Conformal transformation}

Consider the Minkowski line element in polar coordinates

$d s^{2}=-d \eta^{2}+d r^{2}+r^{2} d \Omega_{(2)}^{2}$,

where $r=R$ is trivially the areal radius. The spatially flat Friedmann-Lemaître-Robertson-Walke (FLRW) line element

$d \tilde{s}^{2}=a^{2}(\eta)\left(-d \eta^{2}+d r^{2}+r^{2} d \Omega_{(2)}^{2}\right)$

where $\eta$ is the conformal time, is manifestly conformally flat. The conformal transformation $d \tilde{s}^{2}=\Omega^{2} d s^{2}$ relating (36) and (37) has conformal factor $\Omega=a(\eta)$, the scale factor of the universe. The FLRW areal radius is $\tilde{R}=a(\eta) r$ and the Hubble parameters in comoving time $t$ (given by $d t=a d \eta$ ) and conformal time $\eta$ are, respectively, $H \equiv \dot{a} / a$ and $\mathcal{H}=a_{\eta} / a=a H$ (an overdot denoting differentiation with respect to $t$ ).

To write the FLRW line element (37) in Schwarzschildlike coordinates, we introduce the new time $T$ by

$d T=\frac{1}{F}(d t+\beta d \tilde{R})$,

which transforms (37) to

$$
\begin{aligned}
d \tilde{s}^{2}= & -\left(1-H^{2} \tilde{R}^{2}\right) F^{2} d T^{2} \\
& -2 F\left[-\left(1-H^{2} \tilde{R}^{2}\right) \beta+H \tilde{R}\right] d T d \tilde{R} \\
& +\left[-\left(1-H^{2} \tilde{R}^{2}\right) \beta^{2}+2 H \tilde{R} \beta+1\right] d \tilde{R}^{2} \\
& +\tilde{R}^{2} d \Omega_{(2)}^{2} .
\end{aligned}
$$

The choice

$\beta(t, \tilde{R})=\frac{H \tilde{R}}{1-H^{2} \tilde{R}^{2}}$

reduces the line element to the Schwarzschild-like gauge (6) with $\tilde{A}=\left(1-H^{2} \tilde{R}^{2}\right) F^{2}, \tilde{B}=\left(1-H^{2} \tilde{R}^{2}\right)^{-1}$. As a consequence, the expression (16) of the Brown-York mass in spherical symmetry yields

$$
\begin{aligned}
\tilde{M}_{B Y}^{(F L R W)} & =\tilde{R}\left(1-\sqrt{1-H^{2} \tilde{R}^{2}}\right) \\
& =\tilde{R}\left(1-\sqrt{1-\frac{8 \pi}{3} \rho \tilde{R}^{2}}\right) \\
& =\tilde{R}\left(1-\sqrt{1-\frac{2 M_{M S H}^{(F L R W)}}{\tilde{R}}}\right),
\end{aligned}
$$

where $M_{M S H}^{(F L R W)}=H^{2} \tilde{R}^{2} / 2=\frac{4 \pi}{3} \rho \tilde{R}^{3}$ is the MisnerSharp-Hernandez mass of FLRW space and we used the Friedmann equation $H^{2}=8 \pi \rho / 3$, with $\rho$ the energy density of the cosmic fluid. The relation (41) between the two quasilocal masses in this example mirrors that holding in the Schwarzschild geometry

$d s^{2}=-\left(1-\frac{2 m}{r}\right) d t^{2}+\frac{d r^{2}}{1-2 m / r}+r^{2} d \Omega_{(2)}^{2}$

with (constant) mass $m$. The Misner-Sharp-Hernandez mass contained in a sphere of radius $r$ is $M_{M S H}^{(S c h w)}=m$ for any value of $r>2 m$ and the Brown-York mass is

$M_{B Y}^{(S c h w)}=r\left(1-\sqrt{1-\frac{2 m}{r}}\right)$,

and it asymptotes to $m$ as $r \rightarrow+\infty$. However, on the Schwarzschild event horizon $r=2 m$, it is $M_{B Y}^{(S c h w)}=$ $2 M_{M S H}^{(S c h w)}$

\subsection{Kerr-Schild transformation}

As an example of Kerr-Schild transformation, consider the map between the Minkowski geometry (36) and the ReissnerNordström one

$$
\begin{aligned}
d \tilde{s}^{2}= & -\left(1-\frac{2 m}{r}+\frac{q^{2}}{r^{2}}\right) d t^{2} \\
& +\frac{d r^{2}}{1-\frac{2 m}{r}+\frac{q^{2}}{r^{2}}}+r^{2} d \Omega_{(2)}^{2},
\end{aligned}
$$

which corresponds to

$$
\lambda(t, r)=\frac{m}{r}-\frac{q^{2}}{r^{2}}, \quad l^{\mu}=(1,-1,0,0)
$$

and to the time redefinition $t \rightarrow T$ with

$d T=d t-\frac{\left(\frac{2 m}{r}+\frac{q^{2}}{r^{2}}\right)}{1-\frac{2 m}{r}+\frac{q^{2}}{r^{2}}} d r$.

Minkowski space has vanishing Brown-York mass and the transformation property (34) of the Brown-York mass under Kerr-Schild transformations yields

$\bar{M}_{B Y}=r\left(1-\sqrt{1-\frac{2 m}{r}+\frac{q^{2}}{r^{2}}}\right)$,

which coincides with the well known expression calculated directly using Eq. (2).

\section{Conclusions}

There are several reasons to derive the transformation properties of a quasilocal energy under conformal or (generalized) Kerr-Schild transformations. This procedure is part of the tool-building process useful in various areas of theoretical gravity (black hole thermodynamics, analytical 
solutions of GR describing dynamical black holes, spacetime thermodynamics, etc.). The relativity community seems to have concentrated on the Hawking-Hayward/MisnerSharp-Hernandez quasilocal energy (see, however, Refs. [19, $75,76]$ ) but the Brown-York energy is also interesting in principle because it is based on the Hamilton-Jacobi formulation of GR. However, contrary to the Misner-SharpHernandez mass, the Brown-York constructs suffers from a daunting gauge-dependence even in spherical symmetry. For this reason, the comparison of the "new" Brown-York energy after a spacetime mapping with the "old" one is meaningful only after restoring the gauge which is altered by the spacetime mapping. Having done this and having obtained the "new" Brown-York mass in terms of the "old" one and of the geometry, the result cannot be encapsulated in a simple formula analogous to Eqs. (17) or (35) obtained for the Misner-Sharp-Hernandez mass in [51]. From a pragmatic point of view, the Misner-Sharp-Hernandez construct looks definitely more attractive than the Brown-York one.

Acknowledgements We are grateful to a referee for constructing comments. This work is supported, in part, by the Natural Sciences and Engineering Research Council of Canada (Grant no. 2016-03803 to V.F.) and by Bishop's University.

Data Availability Statement This manuscript has no associated data or the data will not be deposited. [Authors' comment: There are no data because this is a purely theoretical work.]

Open Access This article is distributed under the terms of the Creative Commons Attribution 4.0 International License (http://creativecomm ons.org/licenses/by/4.0/), which permits unrestricted use, distribution, and reproduction in any medium, provided you give appropriate credit to the original author(s) and the source, provide a link to the Creative Commons license, and indicate if changes were made. Funded by SCOAP ${ }^{3}$.

\section{References}

1. R.L. Arnowitt, S. Deser, C.W. Misner, The dynamics of general relativity, in Gravitation: An Introduction to Current Research, ed. by L. Witten (Wiley, New York, 1962), pp. 227-265. (reprinted in arXiv:gr-qc/0405109)

2. L.B. Szabados, Living Rev. Relativ. 12, 4 (2009)

3. S. Hawking, J. Math. Phys. (N.Y.) 9, 598 (1968)

4. S.A. Hayward, Phys. Rev. D 49, 831 (1994)

5. C.W. Misner, D.H. Sharp, Phys. Rev. 136, B571 (1964)

6. W.C. Hernandez, C.W. Misner, Astrophys. J. 143, 452 (1966)

7. S.A. Hayward, Class. Quantum Gravity 15, 3147 (1998)

8. R. Di Criscienzo, M. Nadalini, L. Vanzo, S. Zerbini, G. Zoccatelli, Phys. Lett. B 657, 107 (2007)

9. S.A. Hayward, R. Di Criscienzo, L. Vanzo, M. Nadalini, S. Zerbini, Class. Quantum Gravity 26, 062001 (2009)

10. A.B. Nielsen, Gen. Relativ. Gravit. 41, 1539 (2009)

11. R.-G. Cai, L.-M. Cao, Y.-P. Hu, Class. Quantum Gravity 26, 155018 (2009)

12. M. Pielahn, G. Kunstatter, A.B. Nielsen, Phys. Rev. D 84, 104008 (2011)
13. A.B. Nielsen, J.H. Yoon, Class. Quantum Gravity 25, 085010 (2008)

14. A.C. Wall, Phys. Rev. D 85, 024015 (2012)

15. A.B. Nielsen, M. Visser, Class. Quantum Gravity 23, 4637 (2006)

16. G. Abreu, M. Visser, Phys. Rev. D 82, 044027 (2010)

17. R. Di Criscienzo, M. Nadalini, L. Vanzo, S. Zerbini, G. Zoccatelli, Phys. Lett. B 657, 107 (2007)

18. V. Faraoni, A.B. Nielsen, Class. Quantum Gravity 28, 175008 (2011)

19. F.D. Villalba, P. Bargueño, Phys. Rev. D 99, 044021 (2019)

20. T. Jacobson, Phys. Rev. Lett. 75, 1260 (1995)

21. C. Eling, R. Guedens, T. Jacobson, Phys. Rev. Lett. 96, 121301 (2006)

22. S.A. Hayward, S. Mukohyama, M.C. Ashworth, Phys. Lett. A 256 , 347 (1999)

23. S. Mukohyama, S.A. Hayward, Class. Quantum Gravity 17, 2153 (2000)

24. R.-G. Cai, S.P. Kim, J. High Energy Phys. 02, 050 (2005)

25. R.-G. Cai, L.M. Cao, Phys. Rev. D 75, 064008 (2007)

26. A. Sheykhi, B. Wang, R.-G. Cai, Phys. Rev. D 76, 023515 (2007)

27. R.-G. Cai, L.M. Cao, Y.P. Hu, J. High Energy Phys. 08, 090 (2008)

28. Y. Gong, A. Wang, Phys. Rev. Lett. 99, 211301 (2007)

29. S.F. Wu, B. Wang, G.H. Yang, P.M. Zhang, Class. Quantum Gravity 25, 235018 (2008)

30. K. Bamba, C.Q. Geng, Phys. Lett. B 679, 282 (2009)

31. M. Akbar, R.-G. Cai, Phys. Rev. D 75, 084003 (2007)

32. T. Padmanabhan, Class. Quantum Gravity 19, 5387 (2002)

33. T. Padmanabhan, Phys. Rep. 406, 49 (2005)

34. A. Paranjape, S. Sarkar, T. Padmanabhan, Phys. Rev. D 74, 104015 (2006)

35. D. Kothawala, S. Sarkar, T. Padmanabhan, Phys. Lett. B 652, 338 (2007)

36. G. Chirco, H.M. Haggard, A. Riello, C. Rovelli, Phys. Rev. D 90, $044044(2014)$

37. K. Kleidis, N.K. Spyrou, Class. Quantum Gravity 17, 2965 (2000)

38. J. Sultana, C.C. Dyer, Gen. Relativ. Gravit. 37, 1347 (2005)

39. V. Faraoni, Phys. Rev. D 76, 104042 (2007)

40. M.L. McClure, C.C. Dyer, Class. Quantum Gravity 23, 1971 (2006)

41. M.L. McClure, C.C. Dyer, Gen. Relativ. Gravit. 38, 1347 (2006)

42. M.L. McClure, K. Anderson, K. Bardahl. arXiv:0709.3288

43. M.L. McClure, K. Anderson, K. Bardahl, Phys. Rev. D 77, 104008 (2008)

44. M.L. McClure, Cosmological black holes as models of cosmological inhomogeneities, PhD thesis, Univ. of Toronto. (2005). http:// www.astro.utoronto.ca/theses/thesis05.mcclure.pdf. Accessed 12 Aug 2019

45. K. Bhattacharya, B.R. Majhi, Phys. Rev. D 95, 064026 (2017)

46. A. Mandal, S. Samanta, B.R. Majhi, Phys. Rev. D 94, 064069 (2016)

47. K. Bhattacharya, B.R. Majhi, Phys. Rev. D 94, 024033 (2016)

48. B.R. Majhi, Phys. Rev. D 92, 064026 (2015)

49. J. Cosmol, Astropart. Phys. 1405, 014 (2014)

50. V. Faraoni, Cosmological and Black Hole Apparent Horizons (Springer, New York, 2015)

51. V. Faraoni, V. Vitagliano, Phys. Rev. D 89, 064015 (2014)

52. S.A. Hayward, Phys. Rev. D 53, 1938 (1996)

53. A. Prain, V. Vitagliano, V. Faraoni, M. Lapierre-Léonard, Class. Quantum Gravity 33, 145008 (2016)

54. V. Faraoni, Class. Quantum Gravity 33, 015007 (2015)

55. V. Faraoni, J. Côté. arXiv:1907.08055

56. F. Hammad, Class. Quantum Gravity 33, 235016 (2016)

57. J. Int, Mod. Phys. D 25, 1650081 (2016)

58. R.-G. Cai, L.M. Cao, Y.P. Hu, N. Ohta, Phys. Rev. D 80, 104016 (2009)

59. R.-G. Cai, L.M. Cao, Y.P. Hu, S.P. Kim, Phys. Rev. D 78, 124012 (2008) 
60. H. Zhang, Y. Hu, X.Z. Li, Phys. Rev. D 90, 024062 (2014)

61. S.-F. Wu, B. Wang, G.-H. Yang, Nucl. Phys. B 799, 330 (2008)

62. G. Cognola, O. Gorbunova, L. Sebastiani, S. Zerbini, Phys. Rev. D 84, 023515 (2011)

63. H. Maeda, Phys. Rev. D 73, 104004 (2006)

64. H. Maeda, M. Nozawa, Phys. Rev. D 77, 064031 (2008)

65. J.D.E. Creighton, R.B. Mann, Phys. Rev. D 52, 4569 (1995)

66. K.C.K. Chan, J.D.E. Creighton, R.B. Mann, Phys. Rev. D 54, 3892 (1996)

67. S. Bose, D. Lohiya, Phys. Rev. D 59, 044019 (1999)

68. V. Faraoni, M. Lapierre-Léonard, A. Prain, Phys. Rev. D 92, 023511 (2015)

69. V. Faraoni, M. Lapierre-Léonard, A. Prain, J. Cosmol. Astropart. Phys. 10, 013 (2015)
70. V. Faraoni, M. Lapierre-Léonard, Phys. Rev. D 95, 023509 (2017)

71. M. Lapierre-Léonard, V. Faraoni, F. Hammad, Phys. Rev. D 96, 083525 (2017)

72. R.M. Wald, General Relativity (Chicago University Press, Chicago, 1984)

73. J.D. Brown, J.W. York Jr., Phys. Rev. D 47, 1407 (1993)

74. J.D. Brown, S.R. Lau, J.W. York, Ann. Phys. (NY) 297, 175 (2002)

75. M. Blau, B. Rollier, Class. Quantum Gravity 25, 105004 (2008)

76. P.P. Yu, R.R. Caldwell, Gen. Relativ. Gravit. 41, 559 (2009)

77. R.H. Dicke, Phys. Rev. 125, 2163 (1962)

78. H. Saida, T. Harada, H. Maeda, Class. Quantum Gravity 24, 4711 (2007)

79. V. Faraoni, Universe 4, 109 (2018) 